\title{
Reproducibility of radiomic features of pulmonary nodules between low-dose CT and conventional-dose CT
}

\author{
Yufan Gao ${ }^{1}$, Minghui Hua ${ }^{1}$, Jun $\mathrm{Lv}^{1}$, Yanhe $\mathrm{Ma}^{1}$, Yanzhen $\mathrm{Liu}^{1}, \mathrm{Min} \mathrm{Ren}^{2}$, Yaohua Tian ${ }^{3}, \mathrm{Ximing}{ }^{4}$, \\ Hong Zhang ${ }^{1}$
}

${ }^{1}$ Department of Radiology, Chest Hospital, Tianjin University, Tianjin, China; ${ }^{2}$ Tianjin Cardiovascular Institute, Chest Hospital, Tianjin University, Tianjin, China; ${ }^{3}$ Ministry of Education Key Laboratory of Environment and Health, and State Key Laboratory of Environmental Health (Incubating), School of Public Health, Tongii Medical College, Huazhong University of Science and Technology, Wuhan, China; ${ }^{4}$ Department of Cardiovascular Medicine, Chest Hospital, Tianjin University, Tianjin, China

Contributions: (I) Conception and design: H Zhang, Y Gao; (II) Administrative support: H Zhang; (III) Provision of study materials or patients: Y Gao, J Lv; (IV) Collection and assembly of data: Y Gao, Y Liu, J Lv; (V) Data analysis and interpretation: Y Gao, M Ren, Y Ma, Y Tian, M Hua, X Li; (VI) Manuscript writing: All authors; (VII) Final approval of manuscript: All authors.

Correspondence to: Hong Zhang. Department of Radiology, Chest Hospital, Tianjin University, No. 261 Taierzhuang South Road, Jinnan District, Tianjin 300222, China. Email: tjch_zhanghong@163.com.

Background: The reproducibility of radiomic features is essential to lung cancer detection. This study aimed to investigate the reproducibility of radiomic features of pulmonary nodules between low-dose computed tomography (LDCT) and conventional-dose computed tomography (CDCT).

Methods: A total of 105 patients with 119 pulmonary nodules [39 ground-glass nodules (GGNs) and 80 solid nodules] who underwent LDCT and CDCT were retrospectively studied between September 2019 and November 2020. Pulmonary nodules were manually segmented and 1,125 radiomic features (shape, firstorder intensity, texture, wavelet, and Laplacian of the Gaussian features) were extracted from both LDCT and CDCT images. The concordance correlation coefficient (CCC) was used to evaluate the reproducibility of these radiomic features.

Results: Of the 1,125 radiomic features considered, 35.5\% (399 of 1,125) and 41.5\% (467 of 1,125) were reproducible $(\mathrm{CCC} \geq 0.85)$ for $\mathrm{GGNs}$ and solid nodules, respectively. The intensity, texture, and wavelet features of solid nodules were more reproducible than those of GGNs. The mean CCC values for intensity and texture features of solid nodules were of 0.85 and above, whereas the mean values for those of GGNs were of less than 0.85 . After Gaussian kernel $(\sigma=2)$ preprocessing, the CCC of intensity and texture features of GGNs improved from 0.77 to 0.90 , and $84.9 \%$ (79 of 93 ) of the radiomic features were reproducible (mean CCC increase from $0.84 \pm 0.13$ to $0.92 \pm 0.08$ for intensity features, and from $0.75 \pm 0.15$ to $0.89 \pm 0.11$ for texture features). Wavelet features had the lowest CCCs for both GGNs and solid nodules.

Conclusions: The majority of the radiomic feature classes of solid pulmonary nodules have a high level of reproducibility between LDCT and CDCT. However, LDCT should not be used as an alternative to CDCT in the radiomic study of GGNs.

Keywords: Radiomics; low-dose computed tomography (LDCT); pulmonary nodule; dose

Submitted Jun 15, 2021. Accepted for publication Dec 30, 2021; Published online: 11 Jan 2022.

doi: 10.21037/qims-21-609

View this article at: https://dx.doi.org/10.21037/qims-21-609 


\section{Introduction}

In 2020, lung cancer was ranked second in incidence worldwide, with an estimated 2.2 million new cases; however, with an estimated 1.8 million deaths, it ranked first for mortality (1). In recent years, several studies have demonstrated that low-dose computed tomography (LDCT) is an effective tool for early lung cancer detection which can reduce mortality among high-risk individuals (2-4). The lung imaging reporting and data system (lung-RADS) developed by the American College of Radiology (ACR) was designed to guide radiologists in the diagnosis of nodules detected during lung cancer screening using LDCT. However, the image quality of LDCT is inferior to that of conventional-dose computed tomography (CDCT). Because diagnosis is subjectively determined by radiologists relying on their experience and visually identifiable discriminators can be affected by imaging differences, the use of LDCT in lung cancer screening may increase the false positive rate (5). False positives lead to unnecessary medical treatment, which in turn increases the mental pressure, radiation exposure, and financial burden on patients.

The rapid development of radiomics has provided an effective way to improve diagnosis accuracy in that it provides more objective information than visual recognition (6-8). Radiomics is a process designed for high-throughput extraction of a large number of quantitative features from radiological images, and allows for the conversion of digital medical images into mineable high-dimensional data (6). Recent studies have demonstrated that radiomics can play an important role in LDCT screening of early lung cancer, aiding in the differentiation of benign and malignant pulmonary nodules (9-11), as well as the determination of lung adenocarcinoma invasiveness (12) on baseline LDCT scans. However, as stated above, LDCT can increase the false positive rate of radiologists in comparison with CDCT. We therefore questioned whether radiomic analysis was similarly affected by LDCT. The answer to this question of whether the radiomic features extracted from LDCT associated with early lung cancer are consistent with those extracted from CDCT remains unclear. Several studies to date have reported the effects of different radiation doses on the radiomic features of chest phantoms (13-15), and Lo et al. showed that the effects of different radiation doses on the radiomic features of a uniform water phantom differed from those of a nodule (16). The present study aimed to investigate the reproducibility of radiomic features of pulmonary nodules between LDCT and CDCT.

We present the following article in accordance with the
STROBE (Strengthening the Reporting of Observational Studies in Epidemiology) reporting checklist (available at https://qims.amegroups.com/article/view/10.21037/qims21-609/rc).

\section{Methods}

\section{Study population}

This study was conducted in accordance with the Declaration of Helsinki (as revised in 2013) and was approved by the Medical Ethics Committee of Chest Hospital, Tianjin University (No. 2021LW-005). The requirement to obtain written informed consent was waived due to the retrospective nature of the study.

Data from 105 patients with 119 pulmonary nodules, obtained between September 2019 and November 2020, were used in this study.

The inclusion criteria were as follows: (I) the length and diameter of each nodule was between 5.0 and $30.0 \mathrm{~mm}$; (II) LDCT images were obtained during lung cancer screening, and (III) CDCT images were obtained within $24 \mathrm{~h}$ of the LDCT images for further examination of the details of the nodules and lymph node evaluation. The exclusion criteria were as follows: (I) a history of chest surgery, (II) atelectasis, and (III) severe respiratory artifacts on computed tomography (CT) images.

\section{CT acquisition}

CT scans were acquired on a multi-slice CT scanner (Philips Brilliance, iCT 256). The LDCT scan used the iDose4 reconstruction algorithm with a tube voltage of $100 \mathrm{kV}$ and tube current of 30,40 , or $50 \mathrm{~mA}$ according to the body mass index (BMI) of the patient (BMI $<18.5$, $18.5-25$, and $>25 \mathrm{~kg} / \mathrm{m}^{2}$, respectively). The conventionaldose spiral CT scan used a standard reconstruction algorithm with a tube voltage of $120 \mathrm{kV}$ and tube current ranging from 100 to $250 \mathrm{~mA}$ using automated tube current modulation. The other acquisition parameters were as follows: pitch 0.758 ; field of view, $350 \mathrm{~mm} \times$ $350 \mathrm{~mm}$; section thickness, $1.5 \mathrm{~mm}$; interval, $1.5 \mathrm{~mm}$; matrix, 512×512; and rotation time, $500 \mathrm{~ms}$.

Based on the thin-slice CDCT images, two radiologists (with 12 and 18 years of chest radiology experience) classified pulmonary nodules as ground-glass nodules (GGNs) or solid nodules (17) in consensus, using a lung window setting with a window level of -550 Hounsfield units $(\mathrm{HU})$ and a window width of 1,600 HU. GGNs and 
Table 1 Characteristics of the study population

\begin{tabular}{lcc}
\hline Characteristic & GGNs $(n=39)$ & Solid nodules $(n=80)$ \\
\hline Age, years & $64.95 \pm 9.33[50-81]$ & $65.98 \pm 8.39[51-82]$ \\
Sex, $n(\%)$ & & \\
Female & $27(69.2)$ & $48(60.0)$ \\
Male & $12(30.8)$ & $32(40.0)$ \\
CTDlvol, mGy & & $11.84 \pm 2.89$ \\
C group & $11.45 \pm 2.29$ & $1.87 \pm 0.22$ \\
L group & $1.89 \pm 0.20$ &
\end{tabular}

Data are reported as mean \pm SD. Data in parentheses are ranges (age) and percentages (sex). GGN, ground-glass nodule; C-group, conventional-dose computed tomography group; L-group, low-dose computed tomography group; CTDIvol, volume CT dose index.

solid nodules were defined according to the Fleischner Society guidelines (18).

The volume CT dose index (CTDIvol) was measured during scanning to assess the radiation dose.

\section{Segmentation and radiomic analysis of pulmonary nodules}

Radiomic analysis was performed on images using 3D Slicer software (version 4.10.2; National Institutes of Health, Bethesda, MD, USA) with lung window settings. All nodules in each CT image were manually segmented (slice by slice) by a medical bioengineering graduate student (with 1.5 years of learning experience in chest radiology diagnosis) and a chest radiologist (with 10 years of chest radiology experience). The inter-observer reproducibility of segmentation was initially analyzed with 64 randomly chosen nodules that were segmented independently by the graduate student and the radiologist. The rest of the nodules were segmented by the graduate student and then checked by the radiologist to ensure that each segmentation outcome satisfied the boundary of the nodule. In the case of disagreement, the opinion of the chest radiologist was taken.

A total of 1,125 radiomic features from the 5 feature classes of shape, first-order intensity, texture, wavelets, and Laplacian of the Gaussian (LoG) were calculated and extracted automatically using the module "Radiomics" based on Pyradiomics (version 2.2.0; https://pyradiomics. readthedocs.io/). The shape, first-order intensity, and texture feature classes were calculated based on the original images. The texture features included gray-level co- occurrence matrix (GLCM), gray-level difference matrix (GLDM), gray-level size-zone matrix, (GLSZM), graylevel run-length matrix (GLRLM), and neighborhood gray-tone difference matrix (NGTDM). The LoG features were intensity and texture features at different spatial scales derived from images preprocessed using different LoG spatial bandpass filter parameters (Gaussian kernel sizes of 0 , 1.5, 2.0, and 2.5, with a Gaussian kernel size of 0 indicating an unpreprocessed original image). The wavelet features were intensity and texture features calculated on waveletfiltered images generated using eight frequency band combinations [high-low-low (HLL), low-high-low (LHL), low-high-high (LHH), low-low-high (LLH), high-low-high (HLH), high-high-high (HHH), high-high-low (HHL), and low-low-low (LLL)].

\section{Statistical analysis}

All data were expressed as mean \pm standard deviation. The concordance correlation coefficient (CCC), as defined by Lin (19), was used to evaluate the reproducibility of the radiomic features of both GGNs and solid nodules. The CCC (0 to 1$)$ is estimated using the variance components of a mixed effects model (20). Radiomic features with CCC $\geq 0.85$ were considered reproducible (21). To further investigate the reproducibility of features and the distribution of reproducible features, the CCC threshold for reproducibility was changed from 0.85 to 0.80 and 0.90 , respectively. A Wilcoxon test was used to evaluate differences in the reproducibility of radiomic features between the original images and images preprocessed with Gaussian kernels. Data were analyzed using the $\mathrm{R}$ software (version 4.0.4; http://www.r-project.org/). $\mathrm{P}<0.05$ was considered to be statistically significant.

\section{Results}

\section{Clinical characteristics}

A total of 119 nodules, including 39 GGNs and 80 solid nodules, in 105 patients (39 males and 66 females) were analyzed. The mean age of the patients was $65.6 \pm 8.7$ years (range, 50-82 years; Table 1). The radiation dose parameters for LDCT and CDCT are listed in Table 1. The mean CCC for shape features between the two readers was $0.92 \pm 0.09$ $(0.94 \pm 0.06$ for LDCT and $0.90 \pm 0.11$ for CDCT), which indicated favorable inter-observer reproducibility for nodule segmentation. 


\section{Reproducibility of radiomic features between LDCT and CDCT}

Table 2 presents the CCCs between LDCT and CDCT for the radiomic feature classes. The overall CCCs were

Table 2 CCCs between LDCT and CDCT

\begin{tabular}{lcc}
\hline Feature classes & GGNs $(n=39)$ & Solid nodules $(n=80)$ \\
\hline Shape & $0.90 \pm 0.16$ & $0.76 \pm 0.26$ \\
Intensity & $0.84 \pm 0.13$ & $0.88 \pm 0.11$ \\
Texture & $0.75 \pm 0.15$ & $0.86 \pm 0.12$ \\
Wavelet & $0.55 \pm 0.26$ & $0.67 \pm 0.22$ \\
LoG & & \\
0 & $0.77 \pm 0.15$ & $0.86 \pm 0.12$ \\
1.5 & $0.87 \pm 0.11$ & $0.88 \pm 0.11$ \\
2 & $0.90 \pm 0.11$ & $0.87 \pm 0.12$ \\
2.5 & $0.88 \pm 0.16$ & $0.83 \pm 0.17$ \\
ALL & $0.66 \pm 0.27$ & $0.73 \pm 0.22$ \\
\hline
\end{tabular}

Data are reported as mean \pm SD. For the LoG features, 0 indicates no preprocessing, and $1.5,2$, and 2.5 indicate preprocessing of images with Gaussian kernels of $\sigma=1.5,2$, and 2.5, respectively. CCC, concordance correlation coefficient; CDCT, conventional-dose computed tomography; LDCT, lowdose computed tomography; GGN, ground-glass nodule; LoG, Laplacian of the Gaussian.
$0.66 \pm 0.27$ in GGNs and $0.73 \pm 0.22$ in solid nodules. The mean CCCs of intensity and texture features were $\geq 0.85$ in solid nodules, but $<0.85$ in GGNs. The CCCs for wavelet features were the lowest of the feature classes in both GGNs and solid nodules. In terms of nodule type, the radiomic features of solid nodules (except for shape and LoG features) showed higher reproducibility between LDCT and CDCT than those of GGNs.

Using a CCC value of 0.85 as the cut-off for reproducibility, 467 (41.5\%) of 1,125 radiomic features were reproducible for solid nodules, including 8 (57.1\%) of 14 shape features, $13(72.2 \%)$ of 18 intensity features, 53 (70.7\%) of 75 texture features, 197 (26.7\%) of 739 wavelet features, and 196 (70.3\%) of $279 \mathrm{LoG}$ features. For GGNs, 399 (35.5\%) of 1125 radiomic features were reproducible, including $11(78.6 \%)$ of 14 shape features, $11(61.1 \%)$ of 18 intensity features, 15 (20.0\%) of 75 texture features, 142 $(19.2 \%)$ of 739 wavelet features, and 220 (78.9\%) of 279 LoG features (Table 3).

Figure 1 shows the heat maps of CCCs of radiomic features between LDCT and CDCT. For both GGNs and solid nodules, the first-order intensity features of energy, robust mean absolute deviation, mean absolute deviation, total energy, maximum, root mean squared, 90 percentile, entropy and mean all had high CCC values, as did the texture features of denpendenceentropy, denpendencenonuniformity, gldm_graylevelnonuniformity, jointonentropy, glrlm_

Table 3 Number (percentage) of radiomic features meeting the reproducibility criterion according to different CCC thresholds (cut-off values: $0.80,0.85$, and 0.90 )

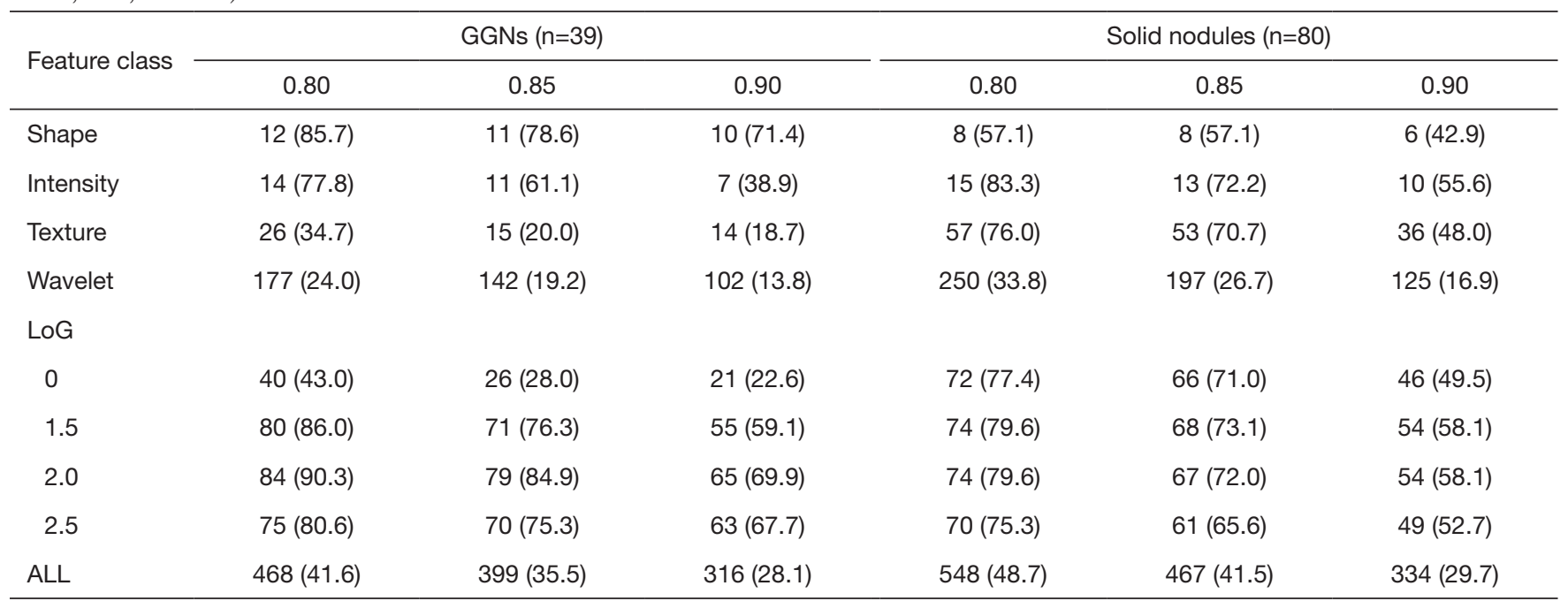

Data in parentheses are percentages. For the LoG features, 0 indicates no preprocessing, and 1.5, 2, and 2.5 indicate preprocessing of images with Gaussian kernels of $\sigma=1.5,2$, and 2.5, respectively. CCC, concordance correlation coefficient; GGN, ground-glass nodule; LoG, Laplacian of the Gaussian. 


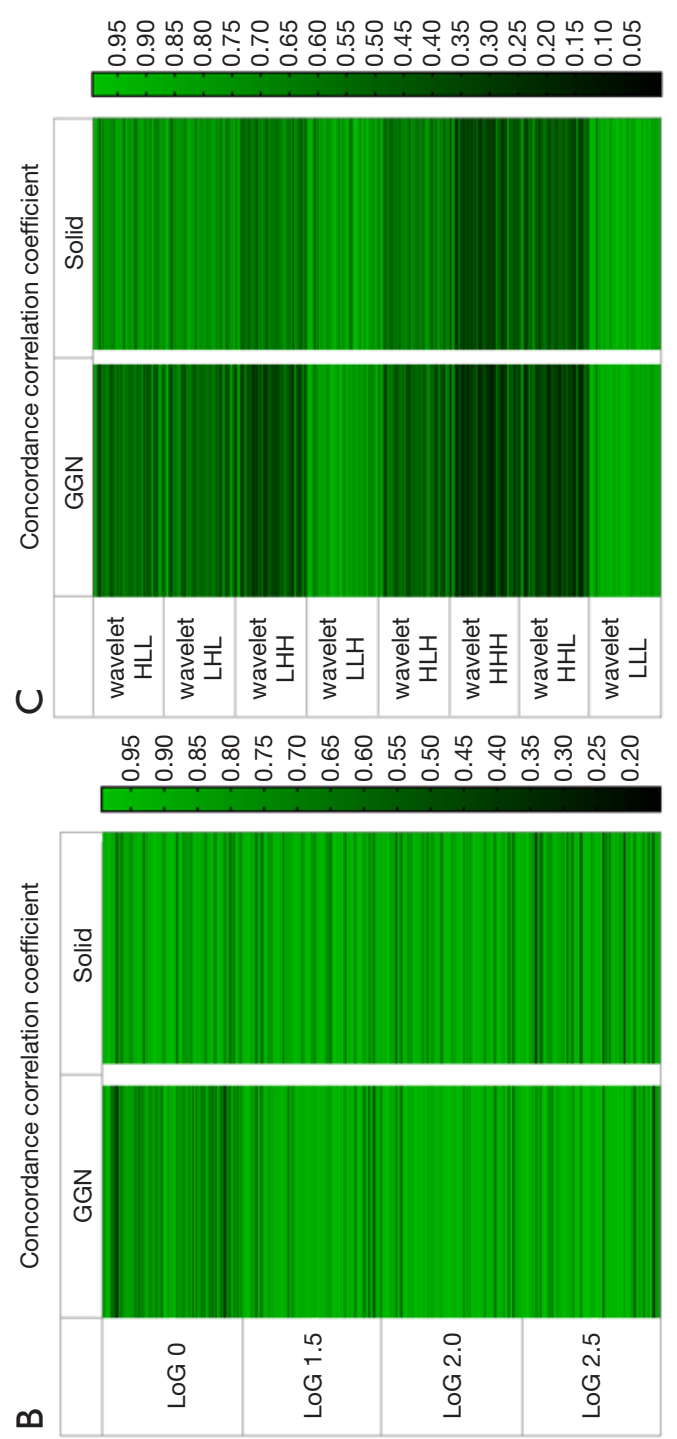

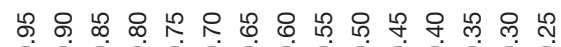

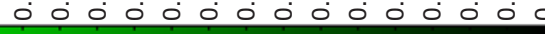

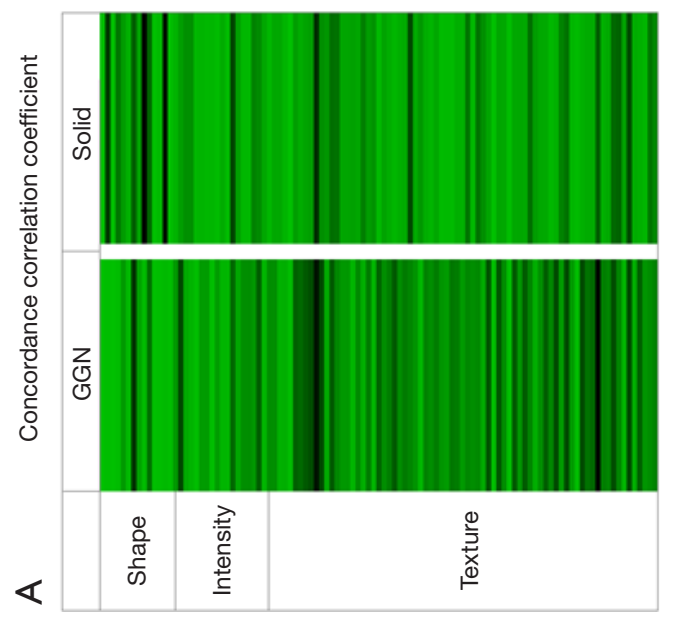

graylevelnonuniformity, runlengthnonuniformity, runentropy, glszm_graylevelnonuniformity, zoneentropy and coarseness (bright colors in Figure 1A), which indicated that they were not sensitive to radiation dose (i.e., image definition) for either of the two nodule types. When CCC $=0.9$ was chosen as the cut-off value for reproducibility, energy, total energy, root mean squared, 90 percentile, denpendencenonuniformity, gldm_graylevelnonuniformity, glrlm_graylevelnonuniformity, runlengthnonuniformity, glszm_graylevelnonuniformity, and coarseness still showed good reproducibility.

\section{Reproducibility of radiomic features after image preprocessing}

The radiomic features of both GGNs and solid nodules showed higher mean CCCs after smoothing with Gaussian kernels of $\sigma=1.5$ and 2. The reproducibility of GGNs was improved more than that of solid nodules, and the overall improvement was more obvious with a Gaussian kernel of $\sigma$ $=2$ than with kernels of $\sigma=1.5$ or 2.5. The CCCs of GGNs increased from $0.77 \pm 0.15$ to $0.90 \pm 0.11$ (first-order intensity features: $0.84 \pm 0.13$ to $0.92 \pm 0.08$; texture features: $0.75 \pm 0.15$ to $0.89 \pm 0.11$ ), and 79 of 93 features $(84.9 \%)$ showed a CCC of $\geq 0.85$, including $16(88.9 \%)$ of the 18 first-order intensity features and $63(84.0 \%)$ of the 75 texture features (Table 2, Figure 2).

For the GGNs, preprocessing with a Gaussian kernel of $\sigma=2$ improved the CCCs of 79 out of 93 radiomic features, by $0.16 \pm 0.12$ on average $(\mathrm{P}<0.001)$, and the CCCs of 53 features increased from below to above the cut-off value of 0.85 . Furthermore, 26 reproducible features extracted from the original images still had a CCC of 0.85 or higher after image processing, although the CCC values of seven features showed slight decreases. After image preprocessing, the CCCs of all features in the GLCM class (except $M C C$, $\mathrm{CCC}=0.83$ ) and the NGTDM class were $>0.85$. The skewness and kurtosis of first-order intensity showed low CCC values for the original images (CCCs of 0.52 and 0.62 , respectively) and the Gaussian kernel-processed images (CCCs of 0.81 and 0.68, respectively). For solid nodules, Gaussian kernel preprocessing improved the mean CCC. However, the CCCs of 10 features decreased to below 0.85 , while those of 53 features improved (the CCCs of 11 features increased from below 0.85 to above 0.85 ). The reproducibility of kurtosis in the original images (CCC $=0.78)$ and Gaussian kernel-processed images $(\mathrm{CCC}=0.70)$ was poor.

For both GGNs and solid nodules, the CCC values 

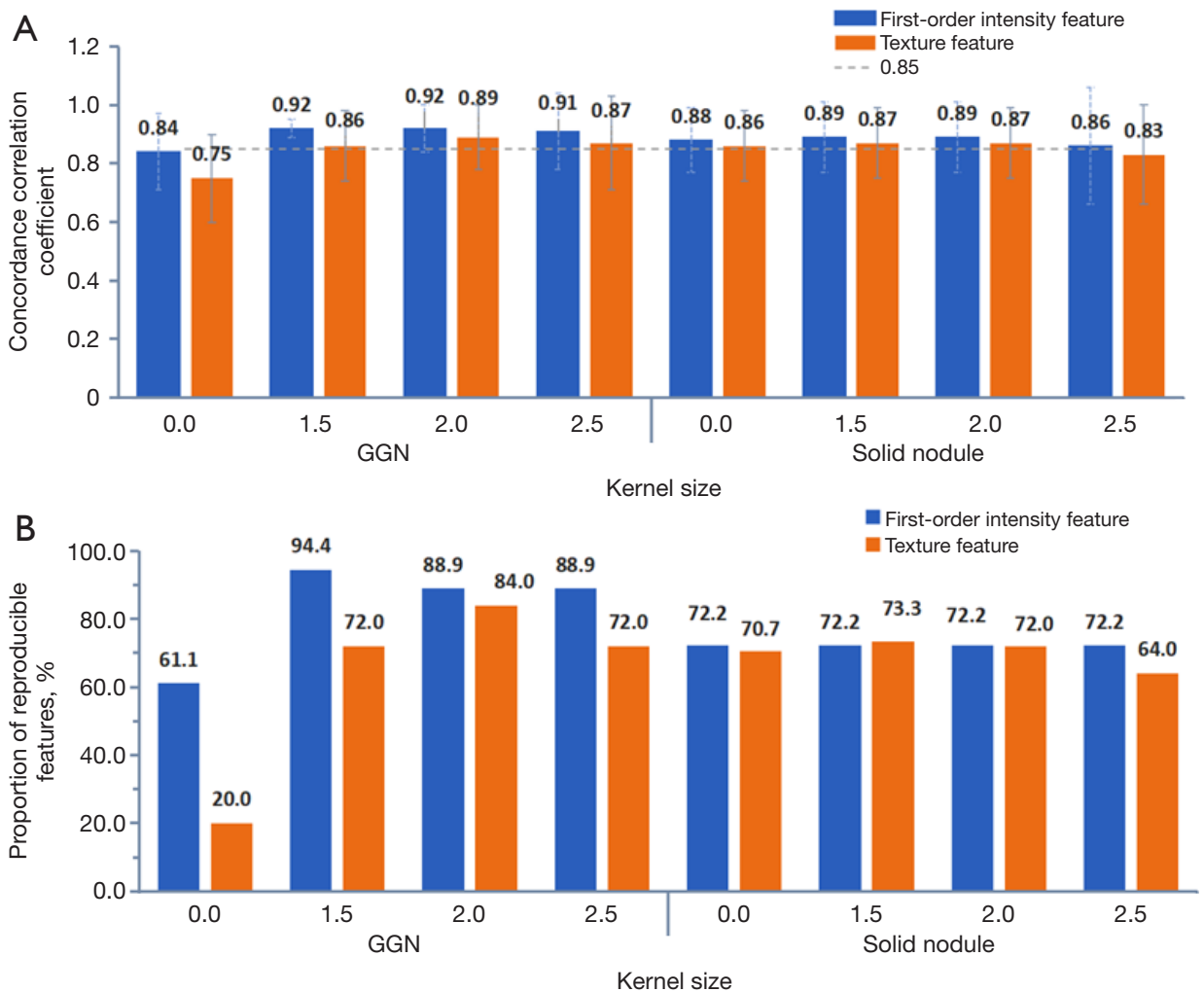

Figure 2 Changes in the reproducibility of intensity and texture features before and after Gaussian preprocessing. (A) CCC values, and (B) proportion of reproducible features $(\mathrm{CCC} \geq 0.85)$, in the ground-glass and solid nodules before and after image preprocessing with different sized Gaussian kernels. CCC, concordance correlation coefficient; GGN, ground-glass nodule.

between LDCT and CDCT were low for wavelet features, and different combinations of high or low frequencies resulted in different CCCs. Compared with solid nodules, the mean CCC of GGNs was lower, and the differences in the CCCs of wavelet features with various high- or lowfrequency combinations were also greater. The CCC values of wavelet features with high-pass filters tended to be lower than those of the other feature types. Wavelet_HHH and wavelet_HHL showed the worst reproducibility (dark colors in Figure 1C). In contrast, wavelet_LLL showed the highest CCC between LDCT and CDCT, followed by wavelet LLH (bright colors in Figure 1C). When a CCC value of 0.85 as the cut-off for reproducibility, wavelet_LLL produced the highest number of reproducible radiomic features (Table 4).

\section{Discussion}

LDCT is currently an important tool in the screening of pulmonary nodules. Radiomics approaches are of value for the differential diagnosis of pulmonary nodules detected using LDCT and for improving diagnostic accuracy. Radiation dose affects CT image quality $(22,23)$, and CDCT can show more details of pulmonary nodules than can LDCT. However, how this difference is reflected in radiomic features remains unclear. The identification of robust quantitative radiomic features was therefore the focus of this study.

Kim et al. reported that radiation dose had a significant influence on the radiomic features of lung nodule phantoms (13). In contrast, Mackin et al.'s study using scans of a phantom acquired with tube currents from 25 to $300 \mathrm{mAs}$ found that the tube current had no significant influence on the radiomic features extracted from CT images of materials with tumor-like textures (14). However, phantom materials are not perfect substitutes for tissue, and some features extracted from pulmonary nodules might have comparatively higher sensitivity to changes in radiation dose.

In this study, most feature classes showed better reproducibility for solid nodules than for GGNs. A possible explanation for this is that the radiation dose affects the 
Table 4 Number (percentage) of reproducible features (CCC $\geq 0.85)$ and CCCs for subclasses of wavelet features

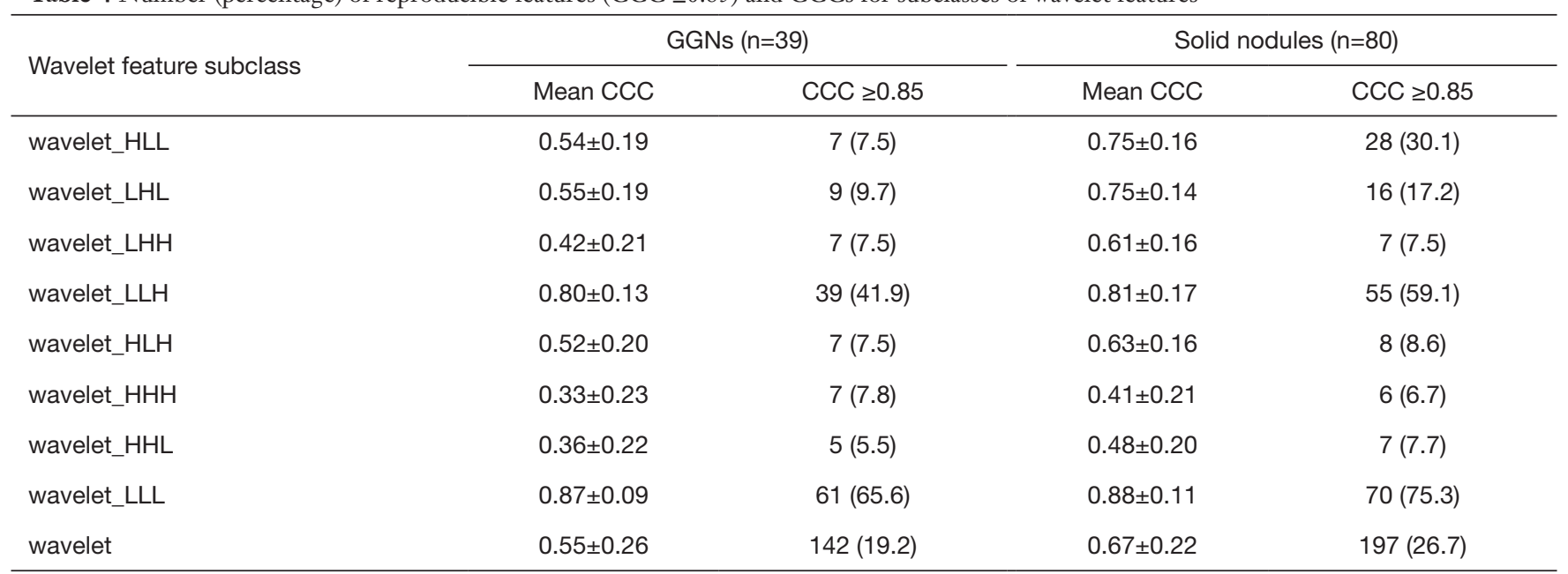

Data are reported as mean \pm SD. Data in parentheses are percentages. CCC, concordance correlation coefficient; GGN, ground-glass nodule; $\mathrm{H}$, high frequency; L, low frequency.

image spatial and density resolution, and because GGNs appear faint, they are more likely to be affected by image resolution. This condition is consistent with traditional visual diagnosis (24). Our results indicate that it may not be appropriate to use LDCT and CDCT interchangeably in the radiomic study of GGNs, as the reproducibility of radiomic features cannot be ensured.

Previous studies have reported that features of different anatomical scales, from fine texture to coarse texture, can be selected by altering the size of the Gaussian filter kernel, thereby allowing tumor heterogeneity to be determined at an appropriate anatomical scale $(25,26)$. In this study, we only reported nodule type, as most of the nodules were concentrated in the 5 to $7 \mathrm{~mm}$ size range. However, nodule size may also impact the reproducibility results, and further study is warranted to explore reproducibility as a function of nodule size. Zhao et al. found that LoG with a Gaussian kernel of $\sigma=2.5$ was highly reproducible for six imaging settings with different slice thicknesses and reconstruction algorithms, and that a smoother reconstruction algorithm was more favorable for reproducibly extracting quantitative features (21). Using a water phantom, Lo et al. showed that feature values extracted from low-dose level images with smooth reconstruction were similar to those extracted from images acquired using other radiation dose and reconstruction algorithm combinations (16). We analyzed the reproducibility of LoG features with $\sigma=1.5,2$, and 2.5, and found that the reproducibility of GGN intensity and texture features improved after images were preprocessed using Gaussian kernels, with a kernel size of 2 performing best (CCC $\geq 0.85,88.9 \%$, and $84.0 \%$ of intensity and texture features, respectively). Gaussian processing is used to smooth the image, and radiomic features tend to be more reproducible when calculated on LDCT and CDCT images that have been smoothed with large Gaussian kernels.

In recent years, wavelet features have come to account for a large proportion of extracted radiomic features. Wavelet features have been reported to aid in reducing the effects of photon noise while enhancing biological heterogeneity detection, thus improving the early prediction of adenocarcinoma $(27,28)$. Furthermore, expression of low-frequency wavelet features was higher in lung adenocarcinoma than in granulomas (29). In the current study, we compared the reproducibility of wavelet features of solid nodules and GGNs, and found that it was generally poor. Wavelet features are sensitive to the differences between LDCT and CDCT because they are extremely susceptible to changes in the spatial and density resolution of images $(21,30)$. We also found differences in the CCCs of wavelet features extracted using different combinations of high- or low-frequencies. Regarding LoG features, wavelet_LLL features extracted from the lowfrequency component (LLL) of the original image (i.e., a smoothed version of the original image) showed good reproducibility between LDCT and CDCT. In contrast, wavelet features extracted with high-pass filters highlighted image details and had comparatively low CCCs, with the wavelet_HHH and wavelet_HHL features showing the 
worst reproducibility. These results are in line with those of previous studies on the effects of CT slice thickness and reconstruction algorithm on the reproducibility of wavelet features of lung nodules (30,31).

In our study, for both GGNs and solid nodules, the reproducibility of the kurtosis of intensity features was poor for both the original images (CCC $=0.62$ and CCC $=0.78$, respectively) and the Gaussian-smoothed images ( $2 \mathrm{~mm}$ kernel, CCC $=0.68$ and $\mathrm{CCC}=0.70$, respectively). Differences in density homogeneity within nodules are reflected in the differences in kurtosis measurements, and the differences in nodule kurtosis between LDCT and CDCT may be related to the different information about nodule heterogeneity provided by the two CT imaging methods. Previous studies have reported that benign and malignant nodules exhibit significant differences in kurtosis, with the kurtosis of malignant nodules being higher than that of benign nodules $(9,32)$. Chae et al. found that high kurtosis was a significant differentiator of preinvasive lesions from invasive pulmonary adenocarcinomas (33).

This study has several limitations. Firstly, the study used manual segmentation, which is time-consuming and presents a greater risk of observer bias than semi- or fullyautomated segmentation. Secondly, three categories of tube current settings were used, rather than automated tube current modulation, which can impact noise, artifacts, and texture. Thirdly, the sample size for this study was not large, especially for GGNs. Finally, due to the absence of pathological evidence, we still do not know which features play a key role in diagnosis. In future studies, we will enlarge the sample size and perform prospective research in combination with a broader range of nodule sizes and pathology results.

In summary, the majority of radiomic feature classes of solid pulmonary nodules show a high level of reproducibility between LDCT and CDCT. LDCT and CDCT should not, however, be used interchangeably in the radiomic study of GGNs. Gaussian smoothing enhances the reproducibility of extracted features.

\section{Acknowledgments}

Thanks to Karl Embleton, PhD, from Liwen Bianji, Edanz Group China (www.liwenbianji.cn/ac), who edited the English text of a draft of this manuscript.

Funding: This work was supported by the Tianjin Science and Technology Plan Project on the construction of data platforms for artificial intelligence-assisted diagnosis of lung nodules and clinical applications of such systems in the diagnosis and treatment of lung cancer (18ZXZNSY00400 to H Zhang).

\section{Footnote}

Reporting Checklist: The authors have completed the STROBE reporting checklist. Available at https://qims. amegroups.com/article/view/10.21037/qims-21-609/rc

Conflicts of Interest: All authors have completed the ICMJE uniform disclosure form (available at https://qims. amegroups.com/article/view/10.21037/qims-21-609/coif). The authors have no conflicts of interest to declare.

Ethical Statement: The authors are accountable for all aspects of the work in ensuring that questions related to the accuracy or integrity of any part of the work are appropriately investigated and resolved. The study was conducted in accordance with the Declaration of Helsinki (as revised in 2013). The study was approved by the Medical Ethics Committee of Chest Hospital, Tianjin University (No. 2021LW-005). Written informed consent was waived due to the retrospective nature of the study.

Open Access Statement: This is an Open Access article distributed in accordance with the Creative Commons Attribution-NonCommercial-NoDerivs 4.0 International License (CC BY-NC-ND 4.0), which permits the noncommercial replication and distribution of the article with the strict proviso that no changes or edits are made and the original work is properly cited (including links to both the formal publication through the relevant DOI and the license). See: https://creativecommons.org/licenses/by-nc-nd/4.0/.

\section{References}

1. Sung H, Ferlay J, Siegel RL, Laversanne M, Soerjomataram I, Jemal A, Bray F. Global Cancer Statistics 2020: GLOBOCAN Estimates of Incidence and Mortality Worldwide for 36 Cancers in 185 Countries. CA Cancer J Clin 2021;71:209-49.

2. Aberle DR, Adams AM, Berg CD, Black WC, Clapp JD, Fagerstrom RM, Gareen IF, Gatsonis C, Marcus PM, Sicks JD. Reduced lung-cancer mortality with lowdose computed tomographic screening. N Engl J Med 2011;365:395-409.

3. Church TR, Black WC, Aberle DR, Berg CD, Clingan 
KL, Duan F, Fagerstrom RM, Gareen IF, Gierada DS, Jones GC, Mahon I, Marcus PM, Sicks JD, Jain A, Baum S. Results of initial low-dose computed tomographic screening for lung cancer. N Engl J Med 2013;368:1980-91.

4. Wilson DO, de Torres JP. Lung cancer screening: how do we make it better? Quant Imaging Med Surg 2020;10:533-6.

5. Moyer VA; U.S. Preventive Services Task Force. Screening for lung cancer: U.S. Preventive Services Task Force recommendation statement. Ann Intern Med 2014;160:330-8.

6. Gillies RJ, Kinahan PE, Hricak H. Radiomics: Images Are More than Pictures, They Are Data. Radiology 2016;278:563-77.

7. Attanasio S, Forte SM, Restante G, Gabelloni M, Guglielmi G, Neri E. Artificial intelligence, radiomics and other horizons in body composition assessment. Quant Imaging Med Surg 2020;10:1650-60.

8. Lee G, Lee HY, Park H, Schiebler ML, van Beek EJR, Ohno Y, Seo JB, Leung A. Radiomics and its emerging role in lung cancer research, imaging biomarkers and clinical management: State of the art. Eur J Radiol 2017;86:297-307.

9. Mao L, Chen H, Liang M, Li K, Gao J, Qin P, Ding X, Li X, Liu X. Quantitative radiomic model for predicting malignancy of small solid pulmonary nodules detected by low-dose CT screening. Quant Imaging Med Surg 2019;9:263-72.

10. Sun Q, Huang Y, Wang J, Zhao S, Zhang L, Tang W, Wu N. Applying CT texture analysis to determine the prognostic value of subsolid nodules detected during lowdose CT screening. Clin Radiol 2019;74:59-66.

11. Garau N, Paganelli C, Summers P, Choi W, Alam S, Lu W, Fanciullo C, Bellomi M, Baroni G, Rampinelli C. External validation of radiomics-based predictive models in lowdose CT screening for early lung cancer diagnosis. Med Phys 2020;47:4125-36.

12. Zhou C, Chan HP, Chughtai A, Hadjiiski LM, Kazerooni EA, Wei J. Pathologic categorization of lung nodules: Radiomic descriptors of CT attenuation distribution patterns of solid and subsolid nodules in low-dose CT. Eur J Radiol 2020;129:109106.

13. Kim YJ, Lee HJ, Kim KG, Lee SH. The Effect of CT Scan Parameters on the Measurement of CT Radiomic Features: A Lung Nodule Phantom Study. Comput Math Methods Med 2019;2019:8790694.

14. Mackin D, Ger R, Dodge C, Fave X, Chi PC, Zhang L, Yang J, Bache S, Dodge C, Jones AK, Court L. Effect of tube current on computed tomography radiomic features. Sci Rep 2018;8:2354.

15. Berenguer R, Pastor-Juan MDR, Canales-Vázquez J, Castro-García M, Villas MV, Mansilla Legorburo F, Sabater S. Radiomics of CT Features May Be Nonreproducible and Redundant: Influence of CT Acquisition Parameters. Radiology 2018;288:407-15.

16. Lo P, Young S, Kim HJ, Brown MS, McNitt-Gray MF. Variability in CT lung-nodule quantification: Effects of dose reduction and reconstruction methods on density and texture based features. Med Phys 2016;43:4854.

17. Naidich DP, Bankier AA, MacMahon H, Schaefer-Prokop CM, Pistolesi M, Goo JM, Macchiarini P, Crapo JD, Herold CJ, Austin JH, Travis WD. Recommendations for the management of subsolid pulmonary nodules detected at CT: a statement from the Fleischner Society. Radiology 2013;266:304-17.

18. Hansell DM, Bankier AA, MacMahon H, McLoud TC, Müller NL, Remy J. Fleischner Society: glossary of terms for thoracic imaging. Radiology 2008;246:697-722.

19. Lin LI. A concordance correlation coefficient to evaluate reproducibility. Biometrics 1989;45:255-68.

20. Carrasco JL, Jover L. Estimating the generalized concordance correlation coefficient through variance components. Biometrics 2003;59:849-58.

21. Zhao B, Tan Y, Tsai WY, Qi J, Xie C, Lu L, Schwartz LH. Reproducibility of radiomics for deciphering tumor phenotype with imaging. Sci Rep 2016;6:23428.

22. Mayo JR, Hartman TE, Lee KS, Primack SL, Vedal S, Müller NL. CT of the chest: minimal tube current required for good image quality with the least radiation dose. AJR Am J Roentgenol 1995;164:603-7.

23. Usman Ali M, Miller J, Peirson L, Fitzpatrick-Lewis D, Kenny M, Sherifali D, Raina P. Screening for lung cancer: A systematic review and meta-analysis. Prev Med 2016;89:301-14.

24. Li F, Sone S, Abe H, MacMahon H, Armato SG 3rd, Doi K. Lung cancers missed at low-dose helical CT screening in a general population: comparison of clinical, histopathologic, and imaging findings. Radiology 2002;225:673-83.

25. Ganeshan B, Panayiotou E, Burnand K, Dizdarevic S, Miles K. Tumour heterogeneity in non-small cell lung carcinoma assessed by CT texture analysis: a potential marker of survival. Eur Radiol 2012;22:796-802.

26. Huang Y, Liu Z, He L, Chen X, Pan D, Ma Z, Liang C, Tian J, Liang C. Radiomics Signature: A Potential Biomarker for the Prediction of Disease-Free Survival 
in Early-Stage (I or II) Non-Small Cell Lung Cancer. Radiology 2016;281:947-57.

27. Chen W, Li M, Mao D, Ge X, Wang J, Tan M, Ma W, Huang X, Lu J, Li C, Hua Y, Wu H. Radiomics signature on CECT as a predictive factor for invasiveness of lung adenocarcinoma manifesting as subcentimeter ground glass nodules. Sci Rep 2021;11:3633.

28. Feng B, Chen X, Chen Y, Liu K, Li K, Liu X, Yao N, Li Z, Li R, Zhang C, Ji J, Long W. Radiomics nomogram for preoperative differentiation of lung tuberculoma from adenocarcinoma in solitary pulmonary solid nodule. Eur J Radiol 2020;128:109022.

29. Beig N, Khorrami M, Alilou M, Prasanna P, Braman N, Orooji M, Rakshit S, Bera K, Rajiah P, Ginsberg J, Donatelli C, Thawani R, Yang M, Jacono F, Tiwari P, Velcheti V, Gilkeson R, Linden P, Madabhushi A. Perinodular and Intranodular Radiomic Features on Lung CT Images Distinguish Adenocarcinomas from Granulomas. Radiology 2019;290:783-92.

Cite this article as: Gao Y, Hua M, Lv J, Ma Y, Liu Y, Ren M, Tian Y, Li X, Zhang H. Reproducibility of radiomic features of pulmonary nodules between low-dose CT and conventionaldose CT. Quant Imaging Med Surg 2022;12(4):2368-2377. doi: 10.21037/qims-21-609
30. Choe J, Lee SM, Do KH, Lee G, Lee JG, Lee SM, Seo JB. Deep Learning-based Image Conversion of CT Reconstruction Kernels Improves Radiomics Reproducibility for Pulmonary Nodules or Masses. Radiology 2019;292:365-73.

31. Park S, Lee SM, Do KH, Lee JG, Bae W, Park H, Jung $\mathrm{KH}$, Seo JB. Deep Learning Algorithm for Reducing CT Slice Thickness: Effect on Reproducibility of Radiomic Features in Lung Cancer. Korean J Radiol 2019;20:1431-40.

32. Kamiya A, Murayama S, Kamiya H, Yamashiro T, Oshiro Y, Tanaka N. Kurtosis and skewness assessments of solid lung nodule density histograms: differentiating malignant from benign nodules on CT. Jpn J Radiol 2014;32:14-21.

33. Chae HD, Park CM, Park SJ, Lee SM, Kim KG, Goo JM. Computerized texture analysis of persistent part-solid ground-glass nodules: differentiation of preinvasive lesions from invasive pulmonary adenocarcinomas. Radiology 2014;273:285-93. 